\title{
Superinfection with difficult-to-treat bacteria in COVID-19 patients: a call for compliance with diagnostic and antimicrobial stewardship
}

\author{
Ombretta Para ${ }^{1} \cdot$ Lorenzo Caruso $^{1} \cdot$ Mattia Ronchetti $^{1} \cdot$ Martina Finocchi $^{1}$ (i) $\cdot$ Stefano Guidi ${ }^{1} \cdot$ Michele Spinicci $^{2}$
}

Received: 13 June 2020 / Accepted: 10 October 2020 / Published online: 22 November 2020

(c) Società Italiana di Medicina Interna (SIMI) 2020

\section{Dear Editor,}

By 24 August 2020, severe acute respiratory syndrome coronavirus (SARS-CoV)-2 has caused over 23 million cases and 800,000 deaths worldwide [1]. Based on the experience from other respiratory viral infections, such as pandemic influenza H1N1, SARS and Middle East respiratory syndrome (MERS), bacterial and/or fungal co-infections are likely to play a major role in increasing mortality [2-4]. Recent data from a systematic review reported a low proportion of pulmonary bacterial co-infection among COVID-19 patients [7\% of all hospitalized patients and $14 \%$ of those admitted to intensive care unit (ICU)] [5]. However, COVID-19 patients with a co-infection are more likely to die than patients who do not have co-infections. A retrospective multicentre cohort study, indeed, found that secondary infections were present in 50\% of non-survivor patients [6].

The World Health Organization (WHO) recommends against indiscriminate use of antibiotics in patients with coronavirus disease 2019 (COVID-19), unless there is clinical suspicion of a bacterial infection [7]. Similarly, the National Institute For Clinical Excellence (NICE) COVID19 guidance recommends to consider antimicrobial use only if a bacterial superinfection is likely and/or for patients with major co-morbidities who are at high risk of severe complications from untreated bacterial infections [8]. However, despite few reports of bacterial co-infection, an overuse of empirical antibiotics prescription in COVID-19 patients has been widely documented [9]. In a recent International Severe Acute Respiratory and Emerging Infections Consortium

Lorenzo Caruso

crslorenzo@gmail.com

1 SOD Medicina Interna 1, Azienda Ospedaliero-Universitaria Careggi, Firenze, Italia

2 SOD Malattie Infettive e Tropicali, Azienda Ospedaliero-Universitaria Careggi, Firenze, Italia
(ISARIC) report, $62 \%$ of patients with COVID-19 had received antimicrobial therapy [10].

In this letter, we describe four cases of bacterial superinfection caused by difficult-to-treat pathogens, occurred in COVID-19 patients, to highlight the importance of a correct diagnostic work-up as a cornerstone to achieve aetiological diagnosis and to promote antimicrobial stewardship principles, also in a pandemic context. Data were retrieved from the electronic folds database of our Internal Medicine ward, in the Careggi University Hospital, a tertiary-care hospital in Florence, Italy, where more than 500 COVID-19 patients have been admitted to date. The four cases reported were observed in the period March 20 - May 1, 2020. In all cases, microbiological diagnosis of bacterial superinfection was achieved by bronchoscopy and bronchoalveolar lavage (BAL) culture, by detecting a bacterial load higher than $10^{3} \mathrm{CFU} / \mathrm{mL}$. Moreover, all the diagnoses were made at least $72 \mathrm{~h}$ after the admission, allowing the definition of healthcare acquired pneumonia (HAP) and/or ventilatorassociated pneumonia (VAP).

The first case was a 77-year-old man affected by hypertension and essential thrombocythemia, hospitalized because of fever and dyspnea, who was diagnosed with COVID-19 through polymerase chain reaction (PCR) positivity on nasopharyngeal swab. On the admission to the ward, severe respiratory failure was observed through arterial blood gas analysis, requiring supplemental oxygen therapy with fraction of inspired oxygen (FiO2) 100\%. Chest X-ray showed multiple opacities with interstitial involvement. Antiviral and immune-modulant treatments with lopinavir-ritonavir, hydroxychloroquine and tocilizumab were administered. Nevertheless, 5 days after hospital admission, the clinical status deteriorated with the necessity of non-invasive ventilation and subsequently orotracheal intubation. A second chest radiography demonstrated a clear worsening of pulmonary parenchyma infiltrates and CT scan confirmed new lung consolidations. At the same time, procalcitonin (PCT) and C-reactive protein (CRP) levels showed a considerable 
increase compared to the admission (from 0.8 to $37 \mathrm{ng} /$ $\mathrm{mL}$ and from 23 to $143 \mathrm{mg} / \mathrm{L}$, respectively). A sample of BAL was obtained and microbiological culture performed, with the identification of Stenotrophomonas maltophilia. Hence, the patient was treated with targeted antibiotic therapy, obtaining a progressive improvement of respiratory exchanges, enough to allow extubation and gradual reduction of need for supplemental oxygen. Finally, the patient was discharged after twenty-seven days of hospital stay.

The second case was a 77-year-old woman with a history of heart failure and mitral-valve biologic prosthesis failure, diabetes mellitus and chronic kidney disease. She was initially hospitalized for acute heart failure associated with community-acquired pneumonia, treated with broadspectrum antibiotic therapy. Clinical course was complicated by severe respiratory failure and atrial fibrillation with high ventricular rate. At this time, SARS-CoV-2 was detected on nasopharyngeal swab and an antiviral therapy was introduced. However, due to respiratory deterioration, non-invasive ventilation was required. Chest CT scan was performed and showed a large consolidation of the lower left lobe. Laboratory test showed a mild alteration of PCT $(28 \mathrm{ng} / \mathrm{mL})$, with increased white blood cells and neutrophilia $\left(18,5 \times 10^{9}\right.$ cells $/ \mathrm{mL}, 84 \%$ neutrophils $)$.

Microbiological culture on BAL specimen revealed a coinfection with a multidrug-resistant (MDR) Acinetobacter baumannii. Unfortunately, the patient died during the fourth day of hospital stay, before the results of BAL culture were available and an appropriate therapy could be introduced.

The third patient was a 61-year-old man with larynx cancer, previously treated with local demolition surgery and subsequent tracheostomy, living in a health care residence because of severe impairment of his autonomy. He was admitted to our hospital for fever and dyspnea. Chest radiography revealed the presence of a single pulmonary opacity, associated with interstitial involvement. Arterial blood gas analysis confirmed mild respiratory failure and PCR on nasopharyngeal swab demonstrated SARS-CoV-2 infection. Although given appropriate medical therapy and supplemental oxygen, clinical conditions abruptly declined and the patient was transferred to the ICU, where he was intubated. Chest radiological reassessment revealed a severe worsening of pulmonary infiltrations and a high PCT $(53 \mathrm{ng} / \mathrm{mL})$. BAL culture was positive for methicillin-resistant Staphylococcus aureus (MRSA) and Corynebacterium striatum. In spite of the introduction of targeted antibiotic therapy, in addition to maximal supportive care, the patient died after eight days in the ICU.

The fourth case was an 80 -year-old man affected by chronic obstructive pulmonary disease, ischemic heart disease, ulcerative rectocolitis and chronic kidney disease. He had a history of four days of fever, dyspnea and diarrhea. On admission, chest radiography showed multiple pulmonary infiltrates, arterial blood gas analysis indicated a moderate respiratory failure and nasopharyngeal swab was negative for COVID-19. Thus, an appropriate supportive medical therapy and supplemental oxygen was administered. A second nasopharyngeal swab was also negative, thereby, considering the high clinical and radiological suspicion of COVID-19, a bronchoscopy was performed. PCR on BAL showed, indeed, a co-infection of SARS-CoV2 and MDR-Escherichia coli. Despite a targeted antibiotic and antiviral therapy, the patient progressive developed refractory hypoxia with increasing PCT levels (up to $40 \mathrm{ng} / \mathrm{mL}$, in comparison with in-range values at admission). He required non-invasive ventilation and was later transferred to the ICU, dying after seventeen days from admission.

Similarly to the patients from our case series, superinfections with difficult-to-treat bacterias, such as MRSA, Enterobacterales and MDR non-fermenting rods, have been reported as a major complication of clinical course of COVID-19 patients [5, 11, 12]. Patients with multiple comorbidities and severe to critical forms of COVID-19 are prone to require prolonged hospitalization, intensive care support and multiple invasive devices, thus being at higher risk of nosocomial infections by difficult-to-treat pathogens. In this context, microbiological diagnosis should always be pursued in case of rapid change in clinical conditions and laboratory or radiological pictures, through adequate respiratory samples taken before introducing broad-spectrum antibiotics [13].

Invasive respiratory sampling, like BAL, increases accuracy of microbiological cultures in patients with hospitalacquired or ventilator-associated pneumonia [14]. However, aerosol-generating procedures should be kept to an absolute minimum in COVID-19 patients due to the risk of airborne transmission and should be performed in an adequately ventilated room, wearing adequate personal protective equipment [15].

In addition to traditional microbiology, diagnostic workup for lower respiratory tract infections may benefit from the implementation of newer multiplex PCR panels, which are able to detect simultaneously the most frequent involved Gram-positive and Gram-negative bacteria and multiple respiratory viruses [16]. However, qualitative results provided by molecular tests require careful interpretation, to distinguish between colonization and infection of the identified agent in the patient airway.

Laboratory tests and radiological imaging can support clinicians in the diagnosis of bacterial superinfection in patients with COVID-19 pneumonia. Some inflammatory biomarkers, like CRP, traditionally used to distinguish bacterial and viral infections, are usually elevated in critically-ill COVID-19 patients, resulting as poorly accurate to drive decisions on antibiotic prescription in this setting [17]. PCT, as a sign of secondary bacterial infection, can timely 
inform the decision of antibacterial agents use for COVID19 patients. Moreover, multiple studies have shown that PCT levels increase in critical or deceased COVID-19 patients, suggesting that bacterial infection may play a crucial role in the aggravation of COVID-19 [18]. Of note, all reported cases in our series showed a PCT increase in concomitance with the onset of bacterial superinfection. On the other hand, thorax CT allows to differentiate the typical infiltrate associated with bacterial lower respiratory tract infection from the typical glass ground opacities seen in COVID-19 patients [19].

In conclusion, despite the unprecedented challenge to the health care systems posed by COVID-19 pandemic, diagnostic and antimicrobial stewardship principles should remain a benchmark in our clinical practice. Antimicrobial resistance is still a major public health threat, which will likely persist after COVID-19, and may even potentially be exacerbated by this pandemic.

\section{Funding None.}

Data availability Further data will be provided on request.

\section{Compliance with ethical standards}

Conflicts of interest The authors declare that they have no confict of interest.

Ethics approval Data collection was approved by the local Ethics Committee. The study was performed in accordance with the ethical principles of the Declaration of Helsinki and with the International Conference on Harmonization Good Clinical Practice guidelines.

Statement of human and animal rights All the procedures performed in this study were in accordance with the ethical standards of the institutionalor national research committee and with the 1964 Helsinki Declaration and its later amendments or comparable ethical standards.

Consent to participate The study was performed as a clinical audit using routine collected clinical data in an anonymised format, and as such is exempt from the need to take specific written informed consent.

Informed consent For this type of study, no informed consent is required.

\section{References}

1. World health organization (2020) Coronavirus disease 2019 (COVID-19): weekly epidemiological update 2. WHO, Geneva

2. McCullers JA (2014) The co-pathogenesis of influenza viruses with bacteria in the lung. Nat Rev Microbiol 12(4):252-262

3. Yap FHY, Gomersall CD, Fung KSC et al (2004) Increase in methicillin-resistant Staphylococcus aureus acquisition rate and change in pathogen pattern associated with an outbreak of severe acute respiratory syndrome. Clin Infect Dis 39(4):511-516

4. Who Mers-Cov Research Group (2013) State of knowledge and data gaps of middle east respiratory syndrome coronavirus (MERS-CoV) in humans. PLoS Curr 5:ecurrents. https://doi.org/10.1371/currents. outbreaks.0bf719e352e7478f8ad85fa30127ddb8

5. Lansbury L, Lim B, Baskaran V, Lim WS (2020) Co-infections in people with COVID-19: a systematic review and meta-analysis. J Infect 81(2):266-275

6. Zhou F, Yu T, Du R, Fan G, Liu Y et al (2020) Clinical course and risk factors for mortality of adult inpatients with COVID19 in Wuhan, China: a retrospective cohort study. Lancet 395(10229): 1038

7. World health organization (2020) Clinical management of COVID19 interim guidance. WHO, Geneva

8. NICE. Managing suspected or confirmed pneumonia COVID-19 rapid guideline: managing suspected or confirmed pneumonia in adults in the community. Guidance 2020. Available at: https://www. nice.org.uk/guidance/ng165/chapter/4-Managing-suspected-or-confi rmed-pneumonia (Last access 24th Aug 2020)

9. Kim D, Quinn J, Pinsky B, Shah NH, Brown I (2020) Rates of coinfection between SARS-CoV-2 and other respiratory pathogens. JAMA 323(20):2085-2086. https://doi.org/10.1001/jama.2020.6266

10. International severe acute respiratory and emerging infection consortium. COVID-19 report. 2020. Available at: https://media.tghn.org/ medialibrary/2020/04/ISARIC_Data_Platform_COVID-19_Repor t_8APR20.pdf. (Last access 24th Aug 2020)

11. Hughes S, Troise O, Donaldson H, Mughal N, Moore LSP (2020) Bacterial and fungal coinfection among hospitalized patients with COVID-19: a retrospective cohort study in a UK secondary-care setting. Clin Microbiol Infect 26(10):1395-1399. https://doi. org/10.1016/j.cmi.2020.06.025

12. Zhang G, Hu C, Luo L et al (2020) Clinical features and short-term outcomes of 221 patients with COVID-19 in Wuhan. China J Clin Virol 127:104364

13. Huttner BD, Catho G, Pano-Pardo JR, Pulcini C, Schouten J (2020) COVID-19: don't neglect antimicrobial stewardship principles!. Clin Microbiol Infect 26(7):808-810. https://doi.org/10.1016/j. cmi.2020.04.024

14. Kalil AC, Metersky ML, Klompas M, Muscedere J, Sweeney DA, Palmer LB, Napolitano LM et al (2016) Management of adults with hospital-acquired and ventilator-associated pneumonia: 2016 clinical practice guidelines by the infectious diseases society of America and the American thoracic society. Clin Infect Dis 63(5):e61-e111

15. Tsilingiris D, Papatheodoridi M, Kapelios CJ (2020) Providing evidence on the ongoing health care workers' mask debate. Intern Emerg Med 15:773-777

16. Lagi F, Pollini S, Zammarchi L (2020) Clinical role of viral identification by a polymerase chain reaction-based diagnostic panel in adults hospitalized with community-acquired pneumonia. Intern Emerg Med 15(4):563-565. https://doi.org/10.1007/s11739-02002282-7

17. Lagi F, Piccica M, Graziani L et al (2020) Early experience of an infectious and tropical diseases unit during the coronavirus disease (COVID-19) pandemic, Florence, Italy, February to March 2020. Euro Surveill 25(17):2000556

18. An PJ, Zhu YZ, Yang LP (2020) Biochemical indicators of coronavirus disease 2019 exacerbation and the clinical implications. Pharmacol Res 159:104946

19. Rubin GD, Ryerson CJ, Haramati LB et al (2020) The role of chest imaging in patient management during the COVID-19 pandemic: a multinational consensus statement from the fleischner society. Chest 158(1):106-116

Publisher's Note Springer Nature remains neutral with regard to jurisdictional claims in published maps and institutional affiliations. 